\title{
ELECTRICAL PRECIPITATION
}

\section{THEORY OF THE REMOVAL OF SUSPENDED MATTER FROM FLUIDS}

\author{
BY W. W. STRONG
}

\section{Abstract of Paper}

The frictional resistance of a small particle moving through a fluid is given by the law of Stokes. Knowing the density and size of the particles and the coefficient of viscosity of the medium, the amount of energy required to remove suspended matter from fluids can be calculated. From the formulas the relative efficiences of the different methods used to remove suspended matter from gases can be obtained. From these formulas the electrical method is shown to be especially adapted to fine suspended particles or to a mixture of gases that can be selectively ionized. In a practical case of electrical precipitation of smoke it is shown that approximately 4 per cent of the energy of the corona discharge is actually expended in the process of precipitation.

The manner of the distribution of the energy of the corona discharge is discussed. The nature of the corona ionization and the "corona" rays is also briefly described.

The application of the theory of electrical precipitation is given so that it is possible to determine the best working conditions for precipitating a given kind of suspended matter from a given fluid. The theory also indicates the limits of usefulness of the method of electrical precipitation.

\section{The Energy Required to Remove Suspended Matter FROM FLUIDS}

IN GENERAL it may be said that the engineering problem of removing suspended smoke, fumes or dust from gases and liquids requires that these particles be given more or less velocity. The gases or liquid usually pass through the precipitation chamber with a certain velocity which we will designate as the longitudinal velocity. If the suspended matter can be given a transverse velocity independently of the velocity of the fluid, we have a way of separating the suspended particles from the fluid. Precipitation is therefore a process of subjecting smoke, fumes or smoke and gases to differential forces. Examples of the action of differential forces are given by " settling" due to

*Manuscript of this paper was received January 7, 1915. 
the gravity forces, the centrifuge process (often applied in flues by the use of sudden changes in the direction of the flue gas current or in fans and centrifuge machines), the separation of suspended matter by the direct action of the electric and magnetic fields, or by the ionization methods as used in the Cottrell and corona methods of electrical precipitation.

In all these methods of precipitating smoke, fumes and dust the amount of energy actually required can be calculated by means of the law of Stokes provided one knows the size (radius of the suspended particles $=a$ ) of the particles, their density $(d)$, their velocity (transverse velocity $V_{t}$ ), the number of particles $(n)$ per unit volume of the medium and the coefficient of viscosity of the medium $(\mu)$.

As a typical example of the precipitation of fumes, smoke or dust let us assume that Fig. 1 represents a cross-section of the precipitation chamber. The length of the chamber is $A B$ or $L \mathrm{~cm}$. and the distance between $A B$ and $C D$ is $l$

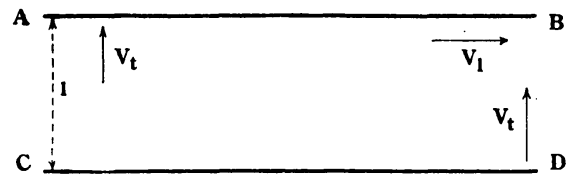

FIG. 1

$\mathrm{cm}$. The longitudinal velocity of the gas through the chamber is $V_{l}$. In order that the fumes shall be precipitated it is necessary that they be given a velocity $V_{t}$ such that they will all be carried to the walls during the time $\left(\frac{V_{l}}{L}\right)$ required for the gases to pass through the precipitation chamber. It will also be assumed that the particles stick to the walls and this is partly accomplished through electrostatic attraction in the electrical method. Complete precipitation for the chamber represented by Fig. 1 therefore requires the transverse velocity $\left(V_{t}\right)$ to carry the particles to either $A B$ or $C D$. We may assume that in one half of the chamber the transverse velocity is towards $A B$ while in the other half it is towards $C D$. (This can be approximated by having an electrically charged plate in the middle of the chamber and charging the smoke particles to an opposite sign.)

We have, therefore, $\frac{1}{2} l / V_{t}=L / V_{l}$ 
Using a margin of safety of 1.2 , we may say $\frac{1}{2} l V_{l}=1.2 \mathrm{~L} V_{t}$ or

$$
V_{l}=\frac{l V_{l}}{2.4 L}=0.4 l V_{l} / L
$$

Assuming our precipitation chamber to be one $\mathrm{cm}$. thick, the volume of gas passing through the chamber per second is $l V_{l} \mathrm{cu}$. $\mathrm{cm}$., and the number of particles passing per second is $n l V_{l}$.

Let $E_{p}$ be the energy required to carry the particles through the gas with the velocity $V_{t}$ and give them their kinetic energy which they eventually transfer to the walls of the chamber when they "stick" to it. Let $F$ be the frictional resistance encountered by the particle and $s$ the distance subjected to this frictional resistance. Then

$$
E_{p}=\left\{\frac{1}{2} m V_{t}^{2}+F s\right\} n \text { ergs per unit. volume. }
$$

For complete precipitation in the chamber of Fig. 1, and for the treatment of $l V_{l} \mathrm{cu} . \mathrm{cm}$. of gas per sec.

$$
E_{p} \text { in ergs per sec. }=n l V_{l}\left(\frac{1}{2} m V_{t}^{2}+\frac{1}{2} F l\right)
$$

The law of Stokes gives the value of the frictional force $F$ and this law has been found to hold with a considerable degree of accuracy for wide variations in the radius, shape and nature of the particles. This law is:

(4) then becomes,

$$
F=6 \pi \mu a V_{t} d .
$$

$$
E_{p} \text { in ergs per sec. }=\frac{1}{2} l V_{l}\left(m V_{t}^{2}+6 \pi \mu a V_{t} d l\right)
$$

or,

$$
E_{p} \text { in ergs per sec. }=\frac{0.2 l^{3} d V_{l}^{2}}{L}\left(\frac{0.16 \pi a^{3} V_{l}}{3 L}+6 \pi \mu a\right)
$$

In the case of smoke and most kinds of fumes and dust $a$ is small. so that the term $\frac{0.16 \pi a^{3} V_{l}}{3 L}$ is negligible. It is in this field that electrical precipitation is especially well adapted, since the forces acting on the smoke particles depend upon the number of ions striking the particle and on the charge of the particle. On the other hand, centrifuge forces vary as the mass of the particles 
and therefore as $a^{3}$. .For centrifuging, equation (7) would remain as stated. For electrical precipitation (7) becomes

$$
E_{p} \text { in ergs per sec. }=\frac{1.2 \pi \mu a l^{3} d V_{l}^{2}}{L}
$$

For practical purposes it is convenient to make the precipitation chamber as large as possible, but it is seen from equation (8) that this is impossible since $l$ appears cubed. It is also seen that $V_{l}$ cannot be increased very greatly even though the length of the precipitation chamber is increased. The greatest effciency is to be attained therefore by making $l$ and $V_{l}$ as small as is practicable. In practical work, $l$ should not exceed 5 or 6 inches, and $V_{l}$ should not greatly exceed 8 or 10 feet per second. The maximum values of $l$ and $V_{l}$ are also limited by the nature of the corona discharge itself, as will be shown later.

\section{- The Corona Discharge}

The corona discharge consists of an electrical current carried by ions produced by the secondary ionization resulting from the impacts of rapidly moving ions with the gas molecules. For purposes of illustration and for practical electrical precipitation of smoke and fumes, the term corona discharge will be restricted to the use of an electrical discharge, taking place between a wire (placed axially) and its enclosing cylinder. The process of secondary ionization is accompanied by a number of transformations. Let $E$ be the energy consumed in the discharge. Part of this energy is converted into the luminous effects of the discharge, $\left(E_{l}\right)$, part in effecting chemical reactions, $\left(E_{c}\right)$, in heating the electrodes, $\left(E_{h \cdot e .}\right)$, in heating the gaseous medium, $\left(E_{h \cdot g}\right)$. We have then,

$$
E=E_{p}+E_{l}+E_{c}+E_{h \cdot e \cdot}+E_{h \cdot g} .
$$

Several of these quantities can be measured separately so that it is to be hoped that our knowledge of these energy changes will soon be greatly extended.

The nature of the ionization taking place during electrical discharges at low gas pressures has been studied by Thomson, Stark and others, and it will be assumed here that the kind and relative number of ions in the "corona rays" and the "corona streams of ions" is the same qualitatively as at low pressures. At atmospheric pressure, however, the ions will be shorter lived, be less simple in constitution and will form less distinct rays, so that the phenomena will not be the same. 
The kind of ions that may exist in oxygen and nitrogen for longer or shorter intervals of time are as follows: $\bar{O}_{2}$, electrons, $\bar{O}, \stackrel{+}{\mathrm{O}}, \stackrel{+}{\mathrm{O}}, \stackrel{+}{\mathrm{O}}_{3}, \stackrel{+}{O}_{8},-$, and charged nuclei. $\stackrel{+}{N}_{2},-, \stackrel{+}{N}_{1},-, \stackrel{+}{N}, \stackrel{+}{N}_{3}$, $+++$

,$- N_{1}$, and charged nuclei. Even at low gas pressures many of these ions appear very rarely, so that one may assume the corona ions to consist largely of $\overline{O_{2}}, \stackrel{+}{N}_{2}, \bar{O}, \stackrel{+}{N}, \stackrel{+}{\mathrm{O}}$, possibly $\stackrel{++}{N}$ and some charged nuclei.

There may be several processes of ionization possible, the process at any place depending on the nature of the gas, the corona rays and the electric field existing there. With increasing field strength the order of appearance of these different types of ionization is probably somewhat as follows:

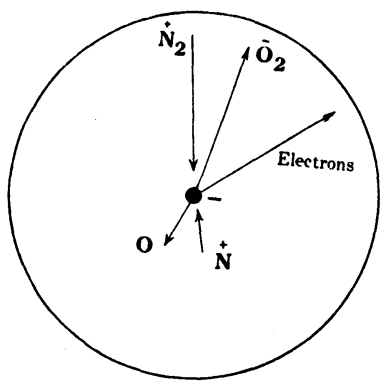

Fig. 2-Negative Corona Rays

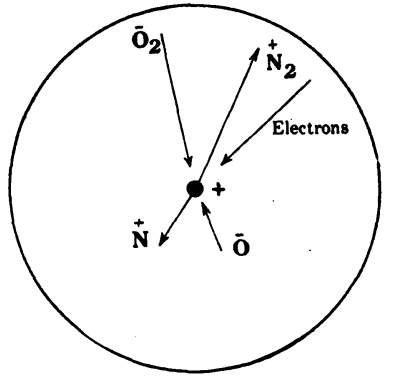

Fig. 3-Positive Corona Rays

(a) Secondary ionization produced by the natural ions of the gas and by the electrons produced by this ionization. The ions are probably almost entirely of the $\bar{O}_{2}$, and $\stackrel{+}{N}_{2}$ type. As the potential gradient (depending on whether there is a + or -corona) is increased, $(b)$, ions like $\bar{O}$ and $\stackrel{+}{N}$, are formed. The different ions probably begin to ionize by collision for different potential gradients, so that the nature of a corona ray depends on the value of this gradient.

The nature of the corona rays is not well known but they are probably somewhat like those represented in Figs. 2 and 3, the positive and negative corona rays being quite different. At low pressures there would be many more electrons, $\stackrel{+}{N}_{2}$ and $\bar{O}_{2}$ rays, than of the other types. The positive brushes may consist of regions where ions recombine. These brushes are very ineffective as regards precipitation, fumes and smoke being almost 
entirely unaffected by them. It seems necessary for precipitation that the suspended particles be subjected directly to the corona rays that are themselves producing secondary ionization. This would lead one to the view that precipitation is not the immediate effect of the electric field on the charge of a smoke particle but that it includes the kinetic energy received by the particle due to collisions with the corona rays.

\section{Best Working Conditions for Electrical Precipitation}

1. The best working conditions for electrical precipitation seems to include the action of as uniform and dense a corona as possible upon the gas containing the suspended matter.

2. The effect of temperature is not very great, higher temperatures usually permitting of a larger corona current density.

3 . In any gas the conditions should be such that no breakdown between the electrodes should take place. Fluctuations in the ionization of the gases may cause considerable trouble.

4. In actual experiments on a precipitation chamber treating about $1000 \mathrm{cu}$. ft. of gas per min. it was found that under the most favorable conditions some 5 per cent of the electrical energy could be converted into $E_{p}$ as given by equation (8). $E_{c}$, the amount of energy used up in chemical reactions such as the oxidation of nitrogen, is of the same order of magnitude.

5 . The efficiency of the process of electrical precipitation is probably not as great as represented by (8) since the corona discharge is not distributed altogether uniformly over the wire.

6. The theory of electrical precipitation can easily be applied to that of a corona wire fixed axially in a cylinder of radius $R$. Consider a cylindrical shell of the thickness $d r$ at a distance $r$ from the center of the cylinder.

The energy required to remove the particles in this shell (volume $=2 \pi r d r L$ ) of number $2 \pi r n L d r$ would be equal to the kinetic energy the particles impart to the cylinder on impact and the energy used up in frictional resistance. One has

$E_{p}$ for this shell per sec. $=$

$$
\left(\frac{1}{2} m V_{r}^{2}+(R-r) 6 \pi \mu a d V_{r}\right) 2 \pi r n L d r,
$$

$(R-r)$ being the distance that each particle must traverse in order to reach the outer cylinder. For the whole cylinder this becomes

$$
E_{p}=\int_{r=b}^{r=R} \frac{1}{2} m V_{r}^{2} d r+12 \pi^{2} r n L(R-r) \mu a d V_{r} d r
$$


in which $b$ is the radius of the wire electrode. Since the particles have reached a state of equilibrium in each shell (the forces of the electric field and the frictional forces being balanced) $V_{r}$ is a function of the potential gradient, this latter being a function of $r ; r$ then is the only variable in (10), so that the expression can be integrated. In this way tables connecting $E_{p}$ with the amount of corona current, the changes in the gas viscosity, temperature, the potential gradient, etc., can easily be determined.

The best method of attacking equation (10) from an experimental point of view is to work with smoke particles of uniform size and density (lycopodium dust for instance), and measure $V_{r}, m, \mu, d$ and $n$. As the law of Stokes has been proved to hold experimentally for wide ranges in the value of $a$ its use permits us to determine the electrical forces that are in more or less equilibrium with the viscous force of resistance. A determination of $V_{r}$ for various values of $r$ will serve to indicate how the smoke particles are ionized. Equation (10) may be written:

$$
E_{p}=A \int_{r=b}^{r=R} V_{r}^{2} d r+B \int_{r=b}^{r=R} r V_{r} d r-C \int_{r=b}^{r=R} r^{2} V_{r} d r .
$$

Since $A$ contains $a^{3}$ and this is very small for fine dust, fumes and smoke, we have for the conditions of electrical precipitation:

$$
E_{p}=B \int_{r=b}^{r=R} r \dot{V}_{r} d r-C \int_{r=b}^{r=R} r^{2} V_{r} d r
$$

The transverse velocity of the ionized smoke particles $\left(V_{r}\right)$ is a function of the potential gradient $\left(E_{r}\right)$ at $r$ and in the direction of $r$.

$$
V_{r}=f\left(E_{r}\right)=f\left(\frac{E}{r \log \frac{R}{b}}\right)
$$

It is thus seen that since $E$ is not a function of $r$, being the difference of potential between the wire and the cylinder, (11) can be integrated. The value of the function $f$ is determined by the size and manner of charging the smoke particles by the corona rays. This is not now well known. We have then for the energy. consumed,

$$
E_{p}=B \int_{r=b}^{r=R} r d r f\left(\frac{E}{r \log \frac{R}{b}}\right)-C \int_{r=b}^{r=R} r^{2} d r f\left(\frac{E}{r \log \frac{R}{b}}\right)
$$


For an example, cigarette smoke was taken. Very dense cigarette smoke may contain a maximum number $(n)$ of $30,000,000$ particles per cu. $\mathrm{cm}$. In the actual experimental conditions $V_{r}$ was assumed uniform and was about $20 \mathrm{~cm}$. per sec. $L=4 \mathrm{ft}$. $a$ was assumed to be $0.01 \mu$ (ranges between 0.1 to $0.001 \mu, \mu$ being a thousandth of a $\mathrm{mm}$.), and the coefficient of viscosity 0.000178 . The particles were all assumed to traverse a distance of $8 \mathrm{~cm}$. The value of $E_{p}$ then comes out to be some 20 to 30 watts (per thousand cu. $\mathrm{ft}$. of smoke) and the corona energy consumption 300 watts. This value of $E_{p}$ is only intended to be a qualitative illustration, but under actual conditions it can be determined with a considerable degree of accuracy.

\section{Conclusions}

1. It is shown that the energy $\left(E_{p}\right)$ required for precipitating smoke, fumes and dust can be represented by the formula

$$
E_{p}=K V l^{2} l^{3}
$$

where $l$ is the length of the precipitation chamber, $V_{l}$ is the necessary longitudinal velocity of the particles and $K$ is a constant that can easily be determined in practise.

2. A formula is derived for the corona method of electrical precipitation that will give the efficiency of the process for pipes of different radii, different lengths and for different values of the potential gradient and the transverse velocity. For installing a plant the problem consists in knowing the constants of this formula and then deriving the most suitable type of precipitation chamber.

3. The possible nature of the corona rays is described, the different kinds of ionization that may take place and the way smoke and fume particles may be charged.

4. The distribution of the energy transformations in the corona discharge is outlined and it is shown how it is possible to measure some of these energy terms.

5. It has been shown that approximately 10 per cent of the energy of the corona discharge may be used directly in precipitaing smoke. There is no doubt but that this efficiency may be increased.

6. It can be shown that a similar percentage of the energy may be used in the oxidation of the nitrogen by the corona discharge. 